$2 \mathrm{KdO}_{3}+\mathrm{Kd} \mathrm{Cl}_{0}+6 \mathrm{H}_{2} \mathrm{O}$.

$2 \mathrm{HgO}+\mathrm{Kd} \mathrm{Cl}_{6}+\mathrm{H}_{2} \mathrm{O}$.

$2 \mathrm{KdO}_{3}+3 \mathrm{KdCl}_{4}$.

$\mathrm{Hg}_{2} \mathrm{O}+\mathrm{KdCl}_{4}$.

VII. Bromrerbindungen.

$\mathrm{Kd} \mathrm{Br}$.

$\mathrm{Hg}_{2} \mathrm{O}+\mathrm{KdBr}_{4}$.

$\mathrm{Kd} \mathrm{Br}_{6}+3 \mathrm{KdO}_{\mathrm{s}}+12 \mathrm{H}_{2} \mathrm{O}$.
VIII. Fluorverbindungen.

$\mathrm{Kd} \mathrm{Fl}_{2}$.

$2 \mathrm{Kd} \mathrm{Fl}+\mathrm{Kd} \mathrm{O}_{3}+3 \mathrm{H}_{2} \mathrm{O}$.

IX. Jodverbindungen.

$\mathrm{KdJ} \mathrm{J}_{2}$.

$\mathrm{KdO}+3 \mathrm{Kd} \mathrm{J}_{2}$.

$\mathrm{X}$. Cyanverbindung.

$\mathrm{Kd} \mathrm{CY}_{\mathbf{2}}$.

\title{
Versuche zur Bestimmung der verschiedenen Natur der Fluorborsäure und der Borfluorwasserstoffsäure ;
}

\author{
von J. Berzelius.
}

(Aus der Köngl. Vet. Acad. Handl. für 1842; übers. von Dr. Wiggers)

Die Fluorborsäure wurde 1810 von Thénard und GayLussac enideckt und in deren Recherches physico-chimiques, T. II. p. 38 beschrieben. Sie wurde erhalten, als sie das ebenfalls von ihnen entdeckte Fluorborgas bis zur völligen Sältigunf; von Wasser absorbiren lielsen. Auf diese Weise bereitet, ist sie cin rauchendes, dickes und schweres Liquidum, aus den durch Kochen eine gewisse Portion Fluorborgas wieder ausgetrieben werden kann; aber darauf raucht die Säure nicht mehr, sondern sie destillirt bei einem hohen Siedpunkte unverändart über. Sie ist dann der concentrirten Schwefelsäure ähnlich, und schwärzt, wie diese, verschiedene organische Stolfe. Gay-Lussac und Thénard geben an, dofs sie mit Basen eigenthümliche Salze bildet, von denen sie jedoch nur das Ammoniumsalz darzustellen versuchten, durch dessen Sublimation (wobei Borsäure zurückblieb) es ihnen glückte, diə Zusammenset- 
zung dieser Säure darzulegen, nach den Ansichten der Zeit, aus Borsăure und aus Flússpathsảure, betrachtet als Sauerstoffsäuren.

Bei den Versuchen, welche ich 1824 der Königl. Acad. über verschiedene Fluorverbindungen mittheilte, zeigte es sich, dars das Fluorborgas, wenn es von Wasser absorbirt wird, ohne dafs dasselbe auf einen höheren Sättigungsgrad gebracht wird, Borsäure absetzt, theils pulverformig und theils beim Erkalten der durch die Absorption des Gases erhitzten Flüssigkeit in Krystallen.

Seitdem sich die chemischen Begriffe von der Yatur der Salzbilder festgesteilt batten, glauben wir zu wissen, dafs das Fluorborgas eine Verbindung von Fluor mit Bor ist, welche keinen Sauerstoff enthält, und welche, gleichwie mehrere andere ähnliche Verbindungen, durch Wasser auf die Weise zersetzt werden mufs, dafs sich der Sanerstoff mit dem Radikal und der Wasserstof mit dem Salzbilder vereinigt. Dieses Factum war also keineswegs eigentlich unerwartet, und da dassclue auswies, dafs auf diese Weise Borsäure und Fluorwasserstofsäure entsteben, so zeigte es auch das bis dahin unbekannte Verhalinifs, in welchem Bor und Fluor mit einander verbunden waren. Aber es stellte sich dabei das Ungewöhnliche heraus, dafs nicht die ganze Quantilät von Fluorbor durch das Wasser auf diese Weise zersetzt wurde, sondern nur $1 / 4$ davon, und dafs nach der $\mathrm{Ab}-$ scheidung der Borsäure der Rückstand cine saure Flüssigkeit war, die, init Basen gesättigt, eigenthümliche, sauerstoftreie Salze gab, welche ich Borfluorsalze nannte, zusammengeselzt aus 1 At Fluorür und $1 \mathrm{Al}$. Flıorbor, und von denen ich diejenigen beschrieben habe, welche mit den gewöhnlicheren, starkeren Salzbasen gebildet werden (K. V. Acad. Handl. 1823. p. 68).

In der von mir untersuchien Borfluorwasserstoffsãure war $1 / 4$ ron dem Bor des Fluorbors gegen Wasserstoff ausgewechselt worden, aber in Thénard's und Gay-Lussac's Fluorborsäure ist das Fluorbor scheinbar unzersetzt mit dem Wasser 
vereinigt, abne dals sich Borsaure abgeschieden hat. Sehr natürlich entstehen die Fragen: Was ist diese Säure? Wie verhalten sich ihre mit Basen gebildeten Salze zu den Borfluorsalzen?

Ehe ich zur Beantwortung dieser Fragen übergehe, will ich einige Betrachtungen über den Grad son Sicherheit anstellen, womil die Zusammenselzung des Fluorbors bekannt ist. Die Schwierigkeiten, weicho man zn überwinden gehabt hat, um Kenntuifs von dem Atomgewicht des Bors zu bekommen, sowohl in Rücksicht auf den Sauerstofligehalt der Borsiuure als auch auf die Auzahl von Sauerstoffatomen, welche diese Säure enthäl, sind bekannt. Wir haben aus den darüber angesteilten Versuchen den Schlurs gezogen, dafs sie aus 1 At. Bor tud 3 At. Saucrstofl besteht.

Bekannjich baben wir gewichtige Gründe für the Annahme, dafs die Gase aller Grundstofe in einem bestimmten Volum eine gleiche Anzall von einfachen Atomen enthalten. Damit stimml auch die specifische Warme derselben überein, welche ebenfalls gleich ist, und die Ausnahmen, welche z. B. bei den Gasen des Schwefels und des Phosphors stattinden, beruhen offenbar auf allotropischen Verhältnissen. Denn in allen bekannten gasförmigen Verbindungen des Schwefels und des Phosphors, welche gewogen werden können, fäll das spec. Gew., mit Beobachtung gewöhnlicher und bei analogen Verbindungen stattfindender Condensations-Verhältnisse bei der Vereinigung, vollkommen normal nach dieser Annahme aus, mit den kleinen $\mathbf{A b}$ weichungen, welche der Druck der Atmosphäre auf leicht coërcibele Gase verursacht, und welche für diese Frage ganz vernachlässigł werden künnen. Daraus folgt also, dafs das normale spec. Gewicht eines jeden Grundstoffs in Gasform sich zu dem Atomgewicht desselben verhalten wird, wie sich das spec. Gew des Sauerstoffgases zu dem Atomgewichl desselben verhält, oder wio das spec. Gew. des Wassergases sich zu dem Atomgewicht des- 
selben verhält. Es ist also möglich, das spec. Gew. eines Grumdstoffs in Gasform zu berechnen, welches dersellbe in der Temperatur hat, worin or in Gas verwandelt wird, anch wem es nicht möglich ist, eine solche $W$ aggung anzustellen; und ist das Atomgewicht richtig bckannt, so wird das berechnete Resultat sicherer, als ein directer Wägungsversuch, wenn dieser ausgefübrt werden hann.

Das Bor gehört zu den Kōrpern, dì nicht in Gasiorm erhalten werden künnen; das spec. Gew desselben in Gasform mul's also berechnet werden. Sein Atomrewicht ist 499,153. Das Atomgewicht des Sauerstoffs ist $=100$, und das spec. (iew. desselben ist nach Baron Wrede's Wägung $=1,1052$. Es entsteht also folgende einfache Rechnung: $100: 1,1052=$ 489,153 : 1,50435, welchas das spec. Gew. des Bors in Gasform wird. Auf ähnliche Weise findę man, dafs das spec. Gew. des Fluorgases $=1,2928$ seyn mufs.

Die Borsäure isi zusimnnengesetzt aus $1 \mathrm{Al}$. Bor und $3 \mathrm{At}$. Sauerstoff, und wenn das Fluorbor Jurch Wasser gerade guch in Borsäure und in Fluorwasserstoffsäure zersetzt wird, so mois das Fluorbor aus 1 At. Bor und 6 At oder 3 Aequ. Fluor bestehen. Dumas hat das Gas des Fluorbors geworgen, und das spec. Gew. desselhen $=2,3124$ geiunden. Es murs in Gasfurm bestehen aus:

$$
\begin{aligned}
& 1 \text { Vol. Borgas . . . . . . . }=1.50435 \\
& 6 \% \text { Fiuorat. . . . }=7,7.2689 \\
& \text { zasainmen wiegend }=9,26115 \text {. }
\end{aligned}
$$

Wemn sich diese 7 Vui, in Augenblicko der Yerouigung von $7 \mathrm{zu} 4$ Vol. condensirt baben, einem Condensations-Yerhaitnisse, wolches zu den gewöbnlichsten grehört, wenn in ein Gas eine gröfsere Anzahi von einiachen Volumen eingetzeten i3t, so ist das spec. Gew. des Fluoriorgases $\frac{9,26115}{4}=2,3153$, und 
es besteht aus 1 Vol. Bor und 3 Vol. Fluor, welche 6 Vol. von dem einfachen Fluorgase entsprechen.

Machen wir eine entsprechende Berechnung für das spec. trew. des Chlorborgases, in welchem das spec. Gew. des einen Bestandtheils durch directe Wägung hat controlirt werden können, so hommen wir zu einem eben so befriedigenden Resultat. Dumas hat das spec. Gew. des Chlorborgases durch directo Wägung bestimmt und dasselbe $=4,03532$ gefunden.

$$
\begin{array}{lllllll}
1 & \text { Vol. Borgas . . . . . . . }=1,50435 \\
6 \% & \text { Chlorgas . . . . . . . . }=\frac{14,64204}{16,14639}
\end{array}
$$

condensirl von 7 Vol. auf 4 erhalten wir $\frac{16,14639}{4}=4,03659$.

Aus der Uebereinstimmung dieser beiden ergiebt es sich, dafs das Alomgewicht des Bors einigermaisen nahe dem rich tigen Verhältnisse gefunden seyn mufs ${ }^{*}$ ).

Aus dem Angeführten folgt mit Sicherheit, dars das Fluor. bor aus 1 At. Bor und 3 Doppelatomen oder Aequivalenten Fluor

*) Es verdient bemerkt zo werden, dafs, wenn man dieselben Berechnungen für die gasförmigen Verbindungen des Biesels mit Fluor und mit Chlor ausfuhrt, man eben so gute Uebereinstimmungen erhält. Dumas hat beide gewogen und für das Fluorkiestigas 3.600 und für das Chlorkieselgas 5,939 grefunden. Das naoh dem Atomgewicht berechnete opec. Gew. für das Kieselgas ist $=3,0649$.

$$
\begin{array}{ll}
1 \text { Vol. Kieselgas }=3,0649 & 1 \text { Vol. Kieselgas }=3,0649 \\
6 " \text { Fluorgas }=7,7568 & 6 \% \text { Chlorgas }=14,6420 \\
\hline 10,8217 . &
\end{array}
$$

Aber hier zeigt es sich, dafs die 7 Vol. sich nicht zu 4, sondem zu 3 condensirt haben, denn $\frac{10,8217}{3}=3,6072$ u. $\frac{17,7069}{3}$ $=5,9023$. Das Vol. der Salzhilder ist hier auf die Hälfte condonsirt und das Yolum des Kiesels ist verschwunden. Dieser Unterschied zwischen beiden. wird nicht durch Berechnung von anderen relativen Atoınverbältnissen in den Verbindungen tines derselben oder beider mit Sauerstofi und mit Salcbildern aufgeboben. 
besteht $=\mathrm{BFI}_{6}$, und dafs es deshalb wissenschaftlich Borsuperfuorid genannt werden muls.

Wenn das Borsuperfluorid bei der Auflossung im Wasser bis zur Sättigung keine Borsäure absetzt, oder die, welche anfänglich abgesetzt wird, wieder aufnimml, so dafs in der concentrirten Flüssigkeit keine Borsäure mehr frei entbalten ist, so rnufs diese Flüssigkeit entweder eine Auflösung von unverändertem Borsuperfluorid oder eine Verbindung von Fluorwasserstoffsüure mit Borsäure seyn. Ist sie ganz einfach eine Auflósung von Borsuperfluorid, so murs sie durck Basen zersetzt werden, ist sie eine chemische Verbindung der Fluorwasserstoifsäure mit Borsäure, verbunden mit Wasser, also eine wasserhaltige Doppelsäure, so mufs sie nit Basen ähnliche Verbindungen cingehen, wie mit Wasser, d. h. es mufs Doppelsalze geben, worin 4 At. von einem borsauren Salze mit 3 Atomen Fluorïr der Base verbunden ist, für jedes Atom Borsäure, welches in dem Salze enthalten ist, und es müssen sich diese direct aus einem borsaurem Salze und einem Fluorür hervorbringen lassen.

Ich loste daher 1 Alongewicht $\mathrm{Na} \mathrm{O}, \mathrm{B} \mathrm{O}_{3}$ und 3 Atongewichte Fluornatrium zusammen in siedendem Wasser auf, eben so 1 Atomgew. $\mathrm{NaO}, 2 \mathrm{BO}_{3}$ (Borax) und 6 Alomgew. Fluornatrium. Die Salze waren vor dem Abwägen frisch geglüht worden. Die Lösungen wurden durch Verdunstang in gelinder Wärme der Krystallisation überlassen, wobei sie bis auf den letzten Tropfen in regelmälsigen Krystallen von einerlei Art anschossen, ohne dafs cins von den Salzen besonders auskrystallisirte. Beide Doppelsalze sind durchsichtig und in der Lun unverānderlich, aber sie verlieren krystallwasser und werden weifs, ohne zu zerfallen, wenn sic an einem $+40^{\circ}$ warmen Orte liegen. Die Krystallform von beiden ist nacb Hrn. Wallmarks Untertersuchung gleich, nämlich vierseitige, dem Ansehen nach rechtwinklige Prismen, welche am Ende schief abgestumpn und mit zwei Ecken obgestumpf sind. Sio scheinen dem triklinometri- 
schen odes dem tetartuprismatischen System atwugehöron. Sio reagiren alkalisoh und b!ihon sich ein wenig auf, ene sie schmelzen, aler bedentend weniger, wie die enfachen borsauren Salze. Das yor dem Löthrolr grschmolzevo Salz erstarrt durchsichtig wre Glas.

Das Salz, welches aus $\mathrm{NaO}_{2} \mathrm{BO}_{3}+3 \mathrm{NaF}_{2}$ bestelit, ver!or beim Erhitzen in Platintiegel bis zum Schmelzen $27 \mathrm{pCL}$ an Gewicls, entsprechend der Formel $3 \mathrm{Na} \mathrm{F}_{2}+\mathrm{NaO} \mathrm{O}_{3} \mathrm{BO}+8 \mathrm{H}_{2} \mathrm{O}$, welche nach der Rechnung 27,26 pCt. Wasser ausweist. Dic goschunolzene Mlasse fullte sicb beim langsamen Erkalten mit kleinen milchweiîsen Kürnern, so dafs sie ibre Durchsichtigkeit nur an den dünnen Fändern, welele am schnellsten erstarrten, behielt. Beim Aulüsen in kaltem Wasser bliob eine durchscheinende, körnige Salzanasse ungelüst zurück, welche im Ansehen dein Borfluornatrium glich, abor sie gab sich ais kleine ubgesetzto Krystalle von Fluornatrium zu erkennen, die, wenn sio mit der Flüssigkei, nachdem dieselbe mit einer hinreichenden Quantität Wasser verdünt worden war, gekocht wurden, sich wieder auflōsten und bein Verdunsten das Doppelsalz wieder in klaren Lrystallen hervorbrachien, die mit einer sehr grofsen Anzahl von Flächen versehon waren.

Das Salz $=6 \mathrm{NaF}_{2}+\mathrm{NaO}, 2 \mathrm{BO}_{3}$ verlor beim Glühen 95,8 $\mathrm{pCt}_{\text {, }}$ entsprechend 22 At. Wasser, welche nach der Rechaung $35,94 \mathrm{pCt}$ betragen ${ }^{*}$, entsprochend den Formeln:

$$
\begin{aligned}
& 3 \mathrm{NaF}_{2}+\mathrm{NaO}_{2} \mathrm{BO}_{3}+8 \mathrm{H}_{2} \mathrm{O} . \\
& 3 \mathrm{NaF}_{2}+\mathrm{BO}_{3}, 2 \mathrm{H}_{2} \mathrm{O}+12 \mathrm{H}_{2} \mathrm{O} .
\end{aligned}
$$

Die Vertheilung des Wassers zwischen beiden Verbindundungen ist allerdings arbitrair und kann $10 \mathrm{At}$. in jeder ausma-

*) Dals in diesan Vermohen der Wassergebalt e:a wenig su goring ausgefalien ist, rubrt von der Eigenechaft dieser Salzo her, in trocknor Luft ein wenig Krystallwaceor an der Oberfâche zu verilieren and dadurch trabe au worden. 
chen, wenn es nicht entschieden ist, dafs der Krystallwassergehalt in dieser Verbindung derselbe seyn werde, wie wenn das Salz allein angeschossen ist. Die geschmolzene Mlasse dieses Saizes enthieit auch einige milchweifse Krystalle von Fluornatrium, zwischen denen das Uebrige durchsichtig war. Nach der Wiederauflösung in siedenden Wasser schofs das Salz bein Verdunsten bis auf den letzten Tropfen an, ohne dafs es Borfluornatrium absetzte, und olme Veranlassung zu der Vermuthung darzubieten, dafs beim Glühen des Salzes Fluorbor gebildet worden und weggegangen sey.

Das letzte Glied in dem cben angeführten Salze enthält eine Verbindung von Fluornatrium mil borsaurem $W$ asser, in demselben relativen Verhältnisse, wie zwischen borsaurem Wasser und Borfluorwasserstoffsäure in der oben angeführten Fluorborsäure. Dabei entstand die Frage: Kann diese Verbindung für sich existiren? Zur Ausmittelung dieser Frage löste ich 1 Alomgew. luftrockner krystullisirter Borsäure, $3 \mathrm{H}_{2} \mathrm{O}, \mathrm{BO}_{3}$, und 3 Alomgew. geglüliten Fluornalriums zusammen in siedendem Wasser auf und verdunstete die Lỏsung in gelinder Wärme, worauf dann beim Erkalten und nachher während fortdauernder freiwilliger Verdunstung die Verbindung in lírystallen anschols, welche denen der vorhergehenden Salze ganz ähnlich waren, ohne dafs Fluornatrium oder Borsuure getrennt auskrystallisioten. Aber der Wassergehalt der Yerbindung konnte leider nicht mit Bestimntheit ausgemillelt werden, weil sieh beim Schmelzen des Salzes Borsuperfluorid entwickelte. Das Salz besals nicht die Eigenschaft, in warmer Luft zu verwittern. Die Krystalle gehürten dem rhomboidalen System an.

Im Uebrigen ist es bemerkenswerth, dafs Borate und Fluorüre der Gegenwart von Wasser zu bedürfen scheinen, um sich in Verbindung $\mathrm{zu}$ erhalten, und dals sie im geschmolzenen Zustande durch die Krystallkraft beim Érkalten getrennt werden. 
In Wasser antgelüst, geben sie mit Salzen der Erden und Metalle Niederschiäge, die sich in den meisten Fällen wieder auflösen, bis cine gewisse Quantitat von dem Fällungsmittel hinzugekonmen ist. Wird daun mehr Wasser linzugesetzt, so lōst sich das Gefälte wieder aut, aber gewönnlich mit Zurücklassung einer kleinen Portion von alggeschiedenern Fluorür. Die Auflösung von $\mathrm{NaO}, 2 \mathrm{BO}_{3}+6 \mathrm{NaF}_{2}$ fäll mit Chlorcalcium nur Fluorcalcimn, erkemular sowohl durch seine gelatinöse Beschaffenheit, als auch durch die Eigenschaft desselben, nach dem Waschen und Trocknen mit Schwefelsäure freie Fluorwasserstoffsäure zu entwickeln.

Ich schmolz Borax und Fluorcalcium in dem zur Bildung des Doppelsalzes erforderlichen Verbältnisse und nit einem kleinen Ueherschufs ron Borax zusammen, pulverisirte das Glas und destillirte dasselbe in einer Glasretorte mit einer hinreichenden Menge conccntrirter Schwefelsäure, wobei mit der Säure zugleich freie Borsäure überging. Das Glusgefäfs wurde nicht angegriffen. Die überdestillirte Säure wurde in einem Platintiegel mit verdünnter reiner Fluorwasserstoffsäure vermischt, in der Absicht, die Borsäure zu binden, und dann im Wasserbade zur Austreibung der Fluorwasserstoffsäure verdunsteł; daun wurde sie in dem 'Tiegel mit umgckehrt aufşelegtem Deckel eine gute halbe Stunde lang über der Flamme einer Spirituslampe gekorht, wobei sich ein grofser Theil der Säure in Gestalt eines dicken Rauchs verflüchtigle. Die Säure wurde dann unter einer Glasglocke über concentrirter Schwefelsäure erkalten gelassen. Sio hatte bei $+18^{\circ}$ ein specifisches Gewicht von 1,5842. Yon dieser Säure wurden 10,511 Grammen abgewogen, init Wasser verdünnt und in einen gewogenen Platintiegel auf 75,175 Grammen frisch gegtüten und pulverisirten Bleioxyds geyossen. Dann n urde das Gemisch in gelinder Warme verdunstet, dabei von Zeit zu Zeit ungerüht, bis zur Trockne, und die Mlasse darauf in dem Tiegel goschmolzen. Sie wog nun 81,54 Gramme, und 
hatle also 4,176 Gr. Wasser abgegeben. Sie hatte folglich in 100 Theilen bestanden aus

$$
\begin{aligned}
& \text { Borsupcrfluorid . . . . . 60,27 } \\
& \text { Wasser . . . . . 39,73. }
\end{aligned}
$$

Abet von diesem Wasser war ein Theil Sauerstof in der Borsäure und Wasserstof in der Fluorwasserstofsaure gewesen 1 Atom Borsuperfluorid wiegt 837,606, und dieses war, nach dem eben angegebenen relativen Verhältnisse, mil 544,41 Theilen Wasser verbunden, die, mit einer Abweichung von - 18,0, 5 Alomen Wasser entsprechen, welche 562,4 wiegen. Diese geringo Abweichung, welche sehr leicht ein Beobachtungsfehler seyn konnte, ergab sich bei der Prüfung als von einer schr geringen Portion Schwefelsäure herrührend, die in der angewandten Fluorborsãure enthalten, aber so unbedeutend war, dafs ich eine Wiederholung des Versuchs für überflüssig hielt. Hieraus folg! also, dafs die Fluorborsäure, von 1,5842 specifischem Gewicht aus 1 Atom Borsäure, 3 Doppelatomen Fluorwasserstoffsäure, zu denen 3 Atome Wasser gegangen sind, und 2 Alomen Wasser besteht $=\mathrm{BO}_{3}+3 \mathrm{H}_{2} \mathrm{~F}_{2}+2 \mathrm{H}_{2} \mathrm{O}$. Es ist wahrscheinlich, dafs die concentrirte Säure, wenn sie in ungekehrter Ordnung bereitet wird, so durs man sie mit Borsuperfluorid ühersätligt, und den Ueberschurs davon darch Kochen daraus austreibh, ein höheres specifisches Gewicht besitzt und nur 1 Atom Wasser enthïlt.

Das Resultat dieser Untersuchung bestebt also darin, dafs Thénard's und Ga y - Lussac's Fluorborsäure cine wasserhaltige Doppelsäure ist, zusammengeselzt aus 3 Aequivalenten Fluorwasserstoffsäure und 1 Aequivalent Borsäure, und dafs dio Salze derselben von einer Verbindung zwischen einem Sauerstofkalze und einem Haloidsalzo ausgemacht werden. Dagegen ist die von mir bescliriebene Borfluorwasserstofsäu:e, welche entsteld, wemm die concentrirte Fluorwasserstoffsäure das Borsuperfluoridgas absorbirt, eine gepaarte Fluorwasserstoffsäure, deren Paarling 
Borsuperfluorid ist, zusummengesetzt aus 1 Atoin von jedem, und deren Solze keinen Sauerstoff enthalten, sondern aus 1 Alom Fluorür mit 1 Atom Borsuperfluorid verbunden bestchen. Die hieselfinorwassersloffsāure ist auf dieselbe Weise eine gepanrte Fluorwasserstuffsäure, deren Paarling Kieselsuperflunrid ist, aber sic besteht ans 3 Atomen Fluorwnsserstoflsäuro und 2 Alomen Kiestlsuperfluorid.

\section{Die Wechselwirthschaft; von J. Liebig.}

Die genauesten Lítersuchungen der thierischen Körper Laben dargethan, dafs Jas Blut, die Knochen, die Haare etc, so wie alle Organe, eine gewisse AnzahJ von Mineralsubstanzen enthalten, mit deren Ausschlurs in der Nahrung, ihre Bildung nicht stattindet.

Das Blut enthält Kali und Natron so wie Verbindungen dieser Basen mit Phosphorsäure, die Galle ist reich an Alkalien, die Substanz der Muskeln enthält eine gewisse Menge Schwefel, das Blutroth esthält Eisen, der Hauptbestandtheil der Knochen ist phospliursaurer Kalk, die Nerven - und Gehirnsubstanz enthäl Phosphorsaure und phosphorsaure Alkalien, der Mlagensaft enthält freie Salzsäure.

Wir wissen, dafs die freie Salzsāure des Magensaftes, dafs ein Theil des Natrons im Blute, vom Kochsalz stamml, dafs wir der Verdaung, dem Leben eine Grenze, durch den blorsen Ausschlufs von Kochsalz setzen.

Geben wir einer jungen Taube Weizenkörner (Chossel, Bericht an die Acadenie in Paris, Juni 1842) zar Nahrung, in denen der Hauplbestandtheil ibrer Knochen, der phosphorsaure Kalk fehlt, so sehen wir, wenn sie gehindert ist, den ihr nothwendigen Kalk sich anderwärls zu verschaffen, dofs ihre lino- 